\title{
Urinary Tract Resections as Part of Debulking Surgery for Locally Advanced Endometrial Stromal Sarcomas
}

\author{
NICOLAE BACALBASA ${ }^{1,2,3}$, IRINA BALESCU ${ }^{4}$, LAURA ILIESCU ${ }^{1,5^{*}}$, CAMELIA DIACONU $^{1,6^{*}}$, \\ SIMONA DIMA ${ }^{3}$, MIHAELA VILCU ${ }^{1,7}$ and IULIAN BREZEAN ${ }^{1,7}$ \\ 1 "Carol Davila” University of Medicine and Pharmacy, Bucharest, Romania; \\ ${ }^{2}$ Department of Obstetrics and Gynecology, "I. Cantacuzino” Clinical Hospital, Bucharest, Romania; \\ ${ }^{3}$ Department of Visceral Surgery, "Fundeni” Clinical Institute, Bucharest, Romania; \\ ${ }^{4}$ Department of Surgery, "Ponderas" Academic Hospital, Bucharest, Romania; \\ ${ }^{5}$ Department of Internal Medicine, "Fundeni" Clinical Institute, Bucharest, Romania; \\ ${ }^{6}$ Department of Internal Medicine, Clinical Emergency Hospital of Bucharest, Bucharest, Romania; \\ ${ }^{7}$ Department of Visceral Surgery, "I. Cantacuzino” Clinical Hospital, Bucharest, Romania
}

\begin{abstract}
Background/aim: Although very rare tumors, uterine sarcomas are extremely aggressive gynecologic malignancies being responsible for a significant number of gynecological cancer-related deaths. However, in such cases, it seems that cytoreductive surgery might increase the lifespan if debulking to no residual disease is achieved. Patients and Methods: We present a case series of three patients diagnosed with endometrial stromal sarcomas in which urinary tract resections were needed in order to maximize the debulking effort. Results: In all cases total hysterectomy with bilateral adnexectomy was performed; in the meantime, urinary tract resection was needed due to the local extension of the neoplastic disease and consisted of unilateral ureteral resection followed by reimplantation through ureteroneocystostomy in one case, bilateral ureteral resection en bloc with partial cystectomy and ureteral reimplantation in one case and total cystectomy with bilateral ureterectomy followed by cutaneous ostomy in the third case. Moreover, one case also necessitated rectosigmoidian resection followed by colorectal anastomosis. In all cases no residual disease was encountered at the end of the debulking surgery.
\end{abstract}

This article is freely accessible online.

*These Authors contributed equally to this study.

Correspondence to: Irina Balescu, "Ponderas" Academic Hospital, Nicolae Caramfil 85a Street, Bucharest, Romania. Tel: +40 724077709, e-mail: irina.balescu@ponderas-ah.ro

Key Words: Endometrial stromal sarcomas, ureteral resection, debulking surgery.
Conclusion: Urinary tract resections might be needed in order to maximize the debulking effort in patients presenting endometrial stromal sarcomas.

Uterine sarcomas represent rare gynecologic malignancies accounting for less of $5 \%$ of all uterine body malignant processes, which are associated with an extremely poor outcome due to their capacity to induce the development of early hematogenous metastases. Moreover, due to this aspect these tumors rarely respond to chemotherapy or radiotherapy (1-3). Unfortunately, preoperatively the diagnosis of uterine sarcoma can be hardly established, since this pathology is mostly suspected in cases presenting rapidly growing uterine masses (4). Due to the rarity of this disease, the most appropriate therapeutic strategy has been strongly debated. However, an important benefit in terms of survival is expected if radical surgery to no residual disease can be achieved $(5,6)$.

\section{Patients and Methods}

We present a case series of three patients diagnosed with locally advanced uterine tumors in which urinary tract resections were needed in order to achieve complete cytoreduction. In all cases, the aim of surgery was to obtain no macroscopic visible residual disease. In all cases, the histopathological studies confirmed the presence of endometrial stromal sarcomas.

\section{Results}

The mean age of patients at the time of surgery was 58 years (range $=53-61$ years), the most frequently encountered symptoms being represented by abdominal pain and vaginal bleeding. However, one case had urinary bladder invasion also associated with hematuria. In all cases surgery consisted of total hysterectomy with bilateral adnexectomy, pelvic and 
para-aortic lymph node dissection. In order to achieve an R0 resection, urinary tract resections consisting of unilateral ureteral resection, bilateral ureteral resection and respectively total cystectomy with bilateral ureteral resection were performed. Moreover, in the third case in which total cystectomy was performed, a rectosigmoidian resection was also performed. In all cases the histopathological studies confirmed the presence of endometrial stromal sarcomas as well as negative resection margins. As for the degrees of differentiation, the two patients presenting limited local invasion of the urinary tract were diagnosed with low grade lesions, while the case presenting urinary bladder and rectosigmoidian invasion was diagnosed with a high grade endometrial stromal sarcoma (Figures 1-7). Interestingly, one of the three cases reported a previous history of endometriosis. Therefore, the patient diagnosed with a pelvic mass invading both the urinary bladder and the rectum had a previous history of endometriosis and had been previously submitted to surgery consisting of excision of an ovarian cyst and of a peritoneal nodule involving the Douglas pouch. However, the histopathological studies confirmed at that moment the benignity of the resected specimen.

\section{Discussion}

Sarcomas are usually considered as poor prognostic tumors irrespective of their origin, with poor long-term survival rates (5-12). Endometrial stromal sarcomas develop from the endometrial stromal cells, which exhibit a different pattern of proliferation inducing the apparition of pelvic pain and vaginal bleeding. This histopathological subtype is responsible for up to $15 \%$ of all uterine sarcomas and $0.2 \%$ of all uterine malignancies (10-12). According to their biological behavior and histopathological features, the World Health Organization (WHO) classified in 2014 endometrial stromal sarcomas in three subtypes: low grade lesions - characterized by an indolent clinical behavior, high grade lesions - characterized by a more aggressive course and undifferentiated lesions which are associated with the poorest outcome $(13,14)$.

When it comes to the aggressiveness of this histopathological subtype of uterine sarcomas, it seems that when compared to leiomyosarcoma and mixed Mullerian tumors, endometrial stromal sarcomas tend to be less aggressive and to have a more favorable outcome. In this respect, the attempt of complete cytoreduction is perfectly justified $(10,12,15)$.

Most often, patients diagnosed with endometrial stromal sarcomas present pelvic extension at the level of the surrounding viscera such as the rectum, ovaries or vagina; however, urinary bladder extension has been rarely encountered $(16,17)$. The largest study conducted on the theme of urinary bladder involvement due to endometrial stromal sarcomas was performed by Tian et al. and included six patients. However, among these six cases a single patient

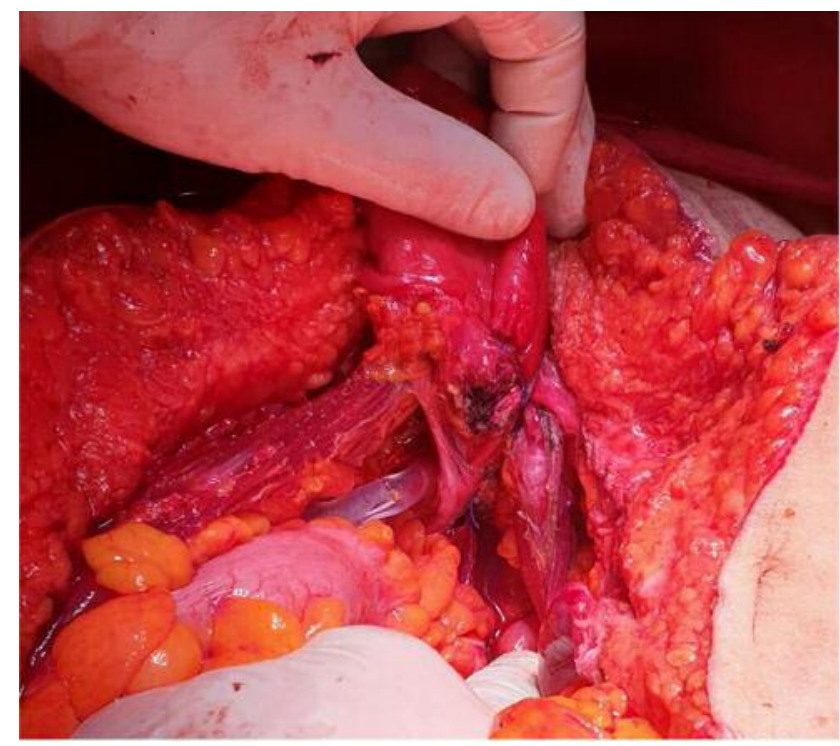

Figure 1. Unilateral ureteral involvement in a patient with locally advanced uterine sarcoma.

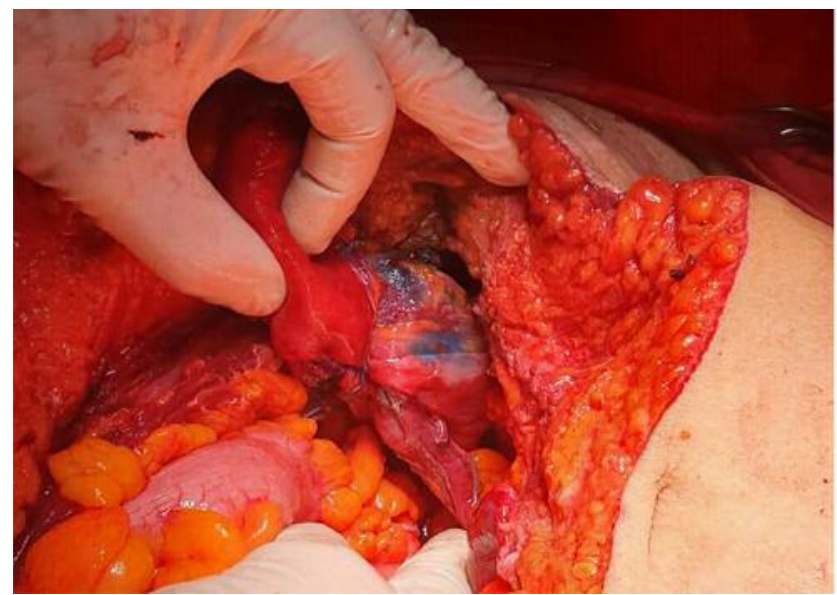

Figure 2. The aspect after right ureteral reimplantation.

presented urinary bladder involvement at the time of the initial diagnosis, the other five patients being diagnosed with pelvic recurrences involving the urinary bladder after a mean interval from the initial diagnosis of 18 years (range $=7-30$ years). As for the type of surgery for urinary bladder involvement, it ranged from transurethral resection of the bladder to partial or total cystectomy and was followed by hormone therapy (18).

When it comes to the efficacy of extended pelvic resections in order to treat locally advanced sarcomas, this aspect has been demonstrated in the study conducted by Waters et al., 


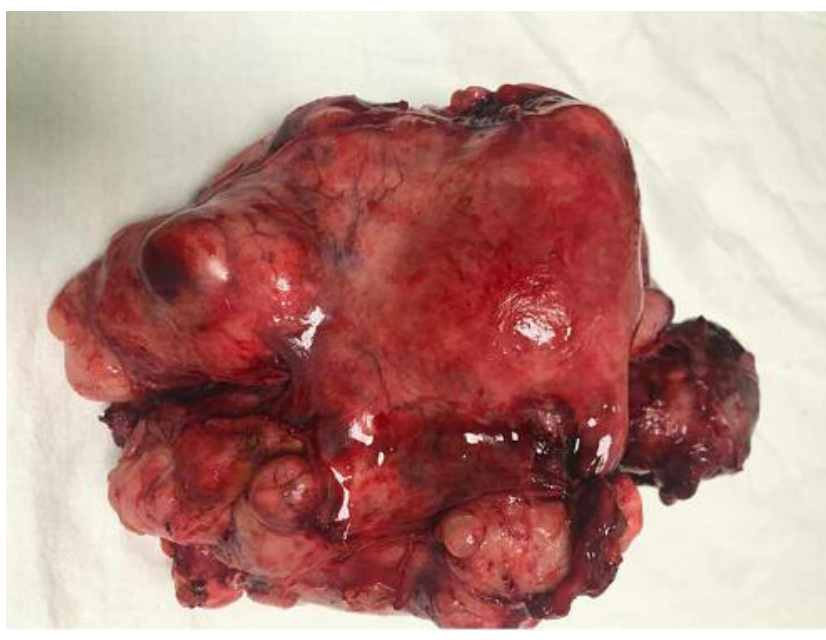

Figure 3. The specimen of total hysterectomy with bilateral adnexectomy and partial cystectomy with unilateral ureteral resection for locally advanced uterine sarcoma.

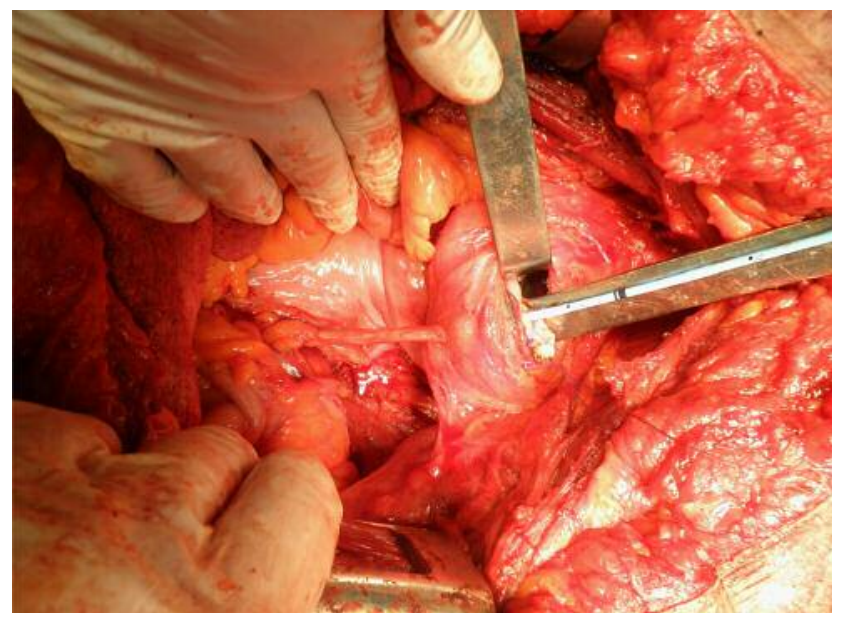

Figure 4. Placing the ureteral stent at the site of uretero-neocystostomy.

which included 670 patients submitted to pelvic exenteration between 1988 and 2018 in two high volume centers (19). Patients submitted to extended pelvic resections for digestive tract malignancies reported a significantly improved long-term survival when compared to those submitted to surgery for gynecological malignancies; however, patients submitted to surgery for sarcomas or for squamous cell carcinomas did not report any difference in terms of survival when compared to those submitted to surgery for adenocarcinomas. An interesting prognostic factor with statistical significance was represented by the moment in which patients were submitted to surgery, where cases included in the last period of time (between 2011 and 2018) reported a significantly longer

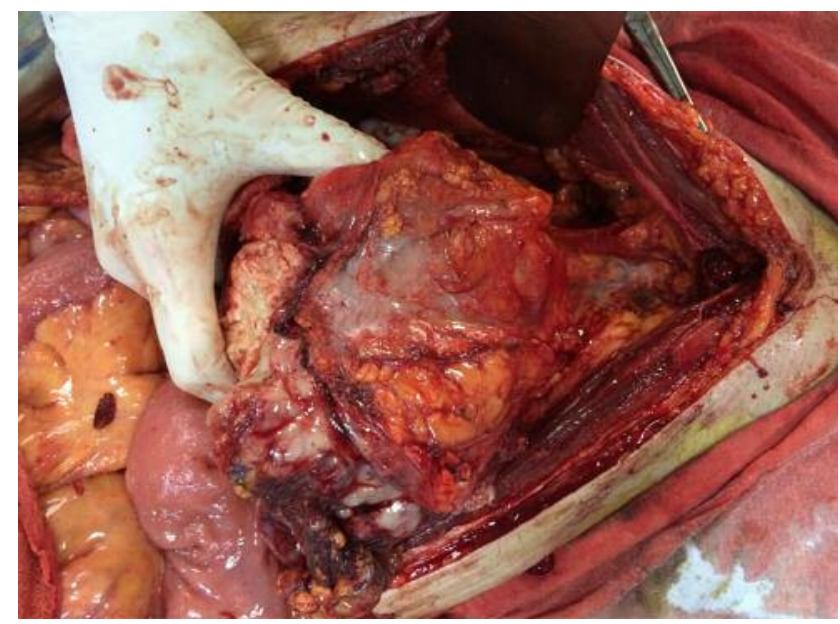

Figure 5. Large uterine body sarcoma invading the urinary bladder and the rectum.

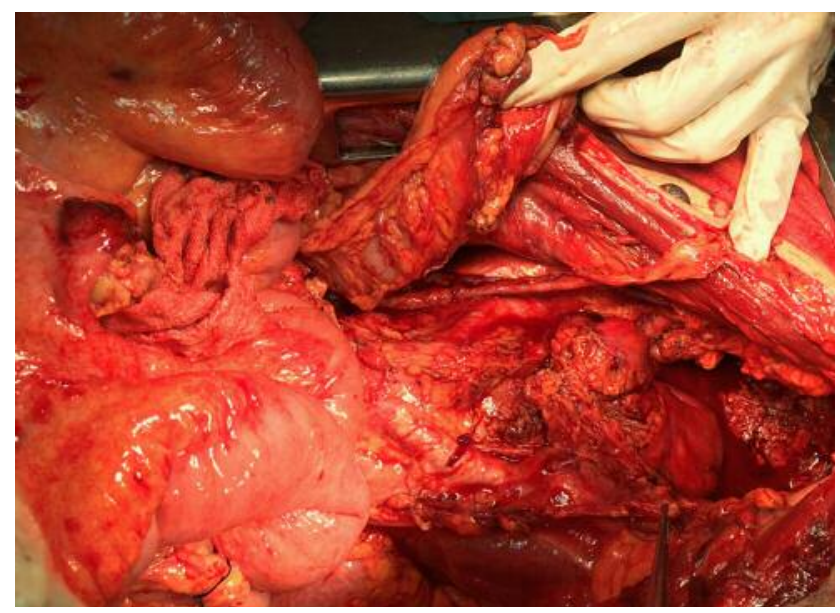

Figure 6. The final aspect after total pelvic exenteration for locally advanced uterine sarcoma.

overall survival when compared to those submitted to surgery between 1988 and 2004 or between 2005 and 2010. Therefore, the authors concluded that technical improvement and learning curve represent important factors influencing the long-term outcomes of these patients irrespective of the origin of the primary tumor (19).

Moreover, in order to maximize the benefits of debulking surgery in cases presenting advanced stage uterine sarcomas with peritoneal involvement, certain authors proposed treatment with hyperthermic intraperitoneal chemotherapy with promising results. Therefore, in the study conducted by Sardi et al. on 36 patients diagnosed with peritoneal sarcomatosis the authors underlined the fact that debulking 


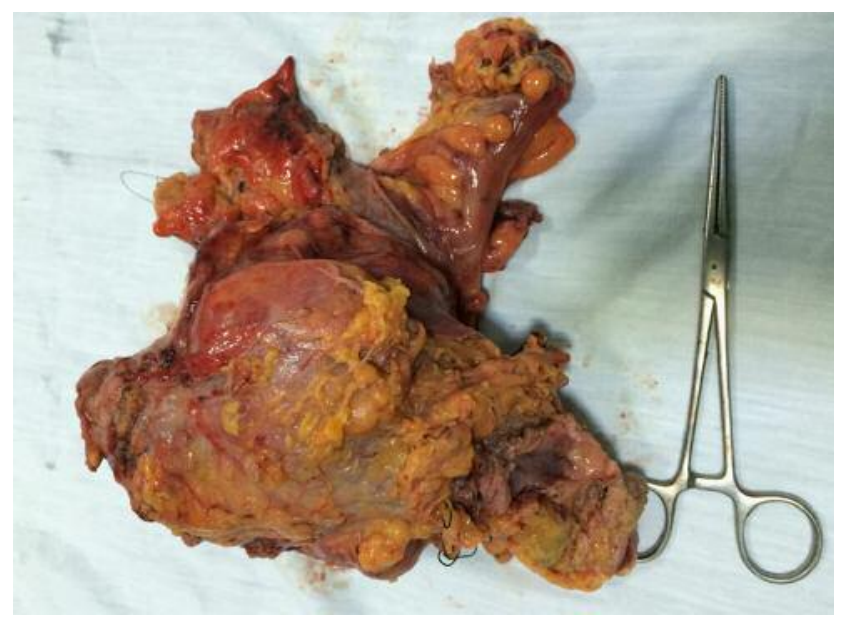

Figure 7. The specimen of total pelvic exenteration for locally advanced uterine sarcoma.

surgery in association with intraperitoneal chemotherapy might improve long-term outcomes especially in cases with uterine leiomyosarcoma; unfortunately the types of associated visceral resections were not detailed, therefore, there are no data regarding the necessity of association of urinary tract resections (20).

As for the malignant transformation of endometriosis, it is a rare complication, described in less than $1 \%$ of cases. Most often this transformation involves the endometriosis foci of the ovaries and leads to the apparition of adenocarcinomas. Endometrial stromal sarcomas in patients with a previous history of endometriosis represent another rare eventuality, only few cases being described so far. Most often, these patients also report a rectosigmoidian invasion, this area being well recognized as a common site of development of deep infiltrating endometriosis (21). Similarly, in our case the patient reported a long history of endometriosis, with previous surgery consisting of resection of an endometrial nodule developed at the level of the Douglas pouch.

In order to maximize the therapeutic effort, multiple strategies have been proposed. However, it seems that debulking surgery to no residual disease seems to have the best results in terms of survival. Moreover, association of adjuvant therapies such as radiation therapy or chemotherapy might improve the rates of 5-year survival especially in patients diagnosed with low grade endometrial stromal sarcomas (21). However, the benefits of the association of chemo-radiotherapy are still strongly debated. Therefore, in the study conducted by Benoit et al. and published in 2005 in including 72 cases of uterine sarcomas, the authors underlined the fact that association of adjuvant treatment does not decrease the risk of metastatic spread, and surgery should still be considered as the therapy of choice. Among the 72 cases the authors included 12 patients with endometrial stromal sarcomas, five of them being treated before 1989 (when adjuvant treatment was not widely used) and the remaining seven cases being treated after 1989. However, no significant differences in terms of rates of recurrences or long-term survival could be observed (22).

\section{Conclusion}

Endometrial stromal sarcomas are rare and aggressive gynecological malignancies with low rates of survival especially if surgery with curative intent is not feasible. However, in cases in which debulking surgery to no residual disease is performed, long-term survival might be encountered in certain cases. In this context, due to the anatomical development of these tumors in the close proximity of the urinary tract, extended resections involving this tract might be needed in order to increase the rates of complete cytoreduction.

\section{Conflicts of Interest}

The Authors have no conflicts of interest to declare regarding this study.

\section{Authors' Contributions}

$\mathrm{NB}, \mathrm{MV}$, IB performed the surgical procedure; IB reviewed literature data; IB, LI, CD prepared the draft of the manuscript; IB was advisor of the surgical oncology procedures; IB reviewed the final version of the manuscript. All Authors read and approved the final version of the manuscript.

\section{Acknowledgements}

This work was supported by the project entitled „Multidisciplinary Consortium for Supporting the Research Skills in Diagnosing, Treating and Identifying Predictive Factors of Malignant Gynecologic Disorders", project number PN-III-P1-1.2-PCCDI2017-0833.

\section{References}

1 Trope CG, Abeler VM and Kristensen GB: Diagnosis and treatment of sarcoma of the uterus. A review. Acta Oncol 51: 694-705, 2012. PMID: 22793037. DOI: 10.3109/028 4186X.2012.689111

2 Gadducci A, Cosio S, Romanini A and Genazzani AR: The management of patients with uterine sarcoma: a debated clinical challenge. Crit Rev Oncol Hematol 65: 129-142, 2008. PMID: 17706430. DOI: 10.1016/j.critrevonc.2007.06.011

3 Nagai T, Takai Y, Akahori T, Ishida H, Hanaoka T, Uotani T, Sato S, Matsunaga S, Baba K and Seki H: Novel uterine sarcoma preoperative diagnosis score predicts the need for surgery in patients presenting with a uterine mass. Springerplus 3: 678, 2014. PMID: 25520907. DOI: 10.1186/2193-1801-3-678 
4 Brezean I, Aldoescu S, Catrina E, Valcu M, Ionut I, Predescu G Degeratu D and Pantea I: Pelvic and abdominal-wall actinomycotic infection by uterus gateway without genital lesions. Chirurgia (Bucur) 105: 123-125, 2010. PMID: 20405693.

5 Bacalbasa N, Balescu I, Vilcu M, Neacsu A, Dima S, Croitoru A and Brezean I: Pelvic exenteration for locally advanced and relapsed pelvic malignancies - an analysis of 100 cases. In Vivo 33 2205-2210, 2019. PMID: 31662557. DOI: 10.21873/invivo.11723

6 Bacalbasa N, Balescu I, Vilcu M, Dima S and Brezean I: The impact of the preoperative status on the short-term outcomes after exenteration and pelvic reconstruction. In Vivo 33: 21472152, 2019. PMID: 31662550. DOI: 10.21873/invivo.11716

7 Bacalbasa N, Taras C, Orban C, Iliescu L, Hurjui I, Hurjui M, Niculescu N, Cristea $M$ and Balescu I: Atypical right hepatectomy for liver metastasis from ovarian leiomyosarcoma a case report and literature review. Anticancer Res 36: 18351840, 2016. PMID: 27069167.

8 Iliescu L, Herlea V, Toma L and Orban C: Association between chronic HCV hepatitis, membranoproliferative glomerulopathy and cutaneous sarcoidosis. J Gastrointestin Liver Dis 24: 8, 2015. PMID: 25822425. DOI: 10.15403/jgld.2014.1121.lil

9 Bacalbasa N, Balescu I, Dima S and Popescu I: Ovarian sarcoma carries a poorer prognosis than ovarian epithelial cancer throughout all FIGO stages: a single-center case-control matched study. Anticancer Res 34: 7303-7308, 2014. PMID: 25503164.

10 Bacalbasa N, Balescu I, Dima S, Brasoveanu V and Popescu I: Prognostic factors and survival in patients treated surgically for primary and recurrent uterine leiomyosarcoma: a single center experience. Anticancer Res 35: 2229-2234, 2015. PMID: 25862883.

11 Dima SO, Bacalbasa N, Eftimie MA and Popescu I: Pancreatic metastases originating from uterine leiomyosarcoma: a case report. World J Surg Oncol 12: 405, 2014. PMID: 25547125. DOI: $10.1186 / 1477-7819-12-405$

12 Bacalbasa N and Balescu I: Atypical paracaval recurrence of uterine endometrial stromal sarcoma: a case report. Anticancer Res 35: 3405-3410, 2015. PMID: 26026102.

13 Hendrickson MR, Tavassoli FA, Kempson RL, McCluggage WG, Haller U and Kubik-Huch RA: Mesenchymal tumours and related lesions. In: Tavassoli FA, Devilee P (eds.). World Health Organization classification of tumours. Pathology and genetics of tumours of the breast and female genital organs. IARC Press, Lyon, pp. 233-244, 2003.

14 Boll D, Verhoeven RH, van der Aa MA, Pauwels P, Karim-Kos HE, Coebergh JW and van Doorn HC: Incidence and survival trends of uncommon corpus uteri malignancies in the Netherlands, 1989-2008. Int J Gynecol Cancer 22: 599-606, 2012. PMID: 22398706. DOI: 10.1097/IGC.0b013e318244cedc
15 Denschlag D, Masoud I, Stanimir G and Gilbert L: Prognostic factors and outcome in women with uterine sarcoma. Eur J Surg Oncol 33: 91-95, 2007. PMID: 17174517. DOI: 10.1016/j.ejso. 2006.11.012

16 Masand RP, Euscher ED, Deavers MT and Malpica A: Endometrioid stromal sarcoma: a clinicopathologic study of 63 cases. Am J Surg Pathol 37: 1635-1647, 2013. PMID: 24121169. DOI: $10.1097 /$ PAS.0000000000000083

17 Yadav S, Santosh M, Bakshi G and Sangeeta D: Extra-uterine low-grade endometrial stromal sarcoma presenting as a urinary bladder mass: a case report with review of the literature. Indian J Surg Oncol, 2019. DOI: 10.1007/s13193-019-00952-3

18 Tian W, Latour M and Epstein JI: Endometrial stromal sarcoma involving the urinary bladder: a study of 6 cases. Am J Surg Pathol 38: 982-989, 2014. PMID: 24705317. DOI: 10.1097/ PAS.0000000000000214

19 Waters PS, Peacock O, Warrier SK, Wakeman C, Eglinton T, Lynch AC, Frizelle FA, Heriot AG and McCormick J: Evolution of pelvic exenteration surgery- resectional trends and survival outcomes over three decades. Eur J Surg Oncol 2019. PMID: 31303376. DOI: 10.1016/j.ejso.2019.07.015

20 Sardi A, Sipok A, Baratti D, Deraco M, Sugarbaker P, Salti G, Yonemura Y, Sammartino P, Glehen O, Bakrin N, Diaz-Montes TP and Gushchin V: Multi-institutional study of peritoneal sarcomatosis from uterine sarcoma treated with cytoreductive surgery and hyperthermic intraperitoneal chemotherapy. Eur J Surg Oncol 43: 2170-2177, 2017. PMID: 28967566. DOI: 10.1016/j.ejso.2017.08.011

21 Chen CW, Ou JJ, Wu CC, Hsiao CW, Cheng MF and Jao SW: High-grade endometrial stromal sarcoma arising from colon endometriosis. Int J Colorectal Dis 22: 1551-1553, 2007. PMID: 17262201. DOI: $10.1007 / \mathrm{s} 00384-006-0267-6$

22 Benoit L, Arnould L, Cheynel N, Goui S, Collin F, Fraisse J and Cuisenier J: The role of surgery and treatment trends in uterine sarcoma. Eur J Surg Oncol 31: 434-442, 2005. PMID: 15837053. DOI: $10.1016 /$ j.ejso.2005.01.010
Received November 26, 2019

Revised December 2, 2019

Accepted December 6, 2019 